| Ciencia, Tecnología y Política | Año 2 | N² | Enero-Junio 2019 | ISSN 2618-3188 | www.revistas.unlp.edu.ar/CTyP |

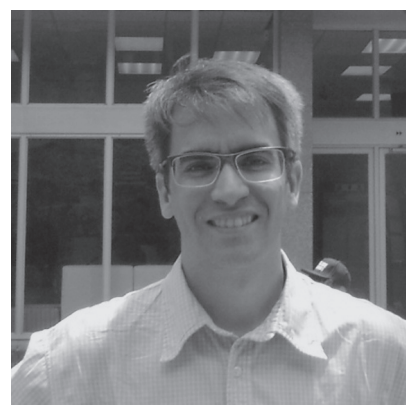

Juan Martín Quiroga

Magíster en Ciencia Tecnología

e Innovación. Centro de Estu-

dios en Ciencia, Tecnología,

Cultura y Desarrollo (CITECDE)

de la UNRN.

jquiroga@unrn.edu.ar

\title{
Producción nacional de radares: expresión de una soberanía tecnológica posible
}

Resumen: Argentina es un país periférico, dependiente de potencias extranjeras en cuestiones ligadas a muchas tecnologías intensivas en conocimiento, pero que a lo largo de su historia ha logrado ser soberano en otras, como por ejemplo en el área nuclear. En el caso del control del tráfico y del espacio aéreo, la soberanía tecnológica se logró, luego de más 50 años de aprendizajes en un contexto de dependencia, y gracias a la interacción entre la Fuerza Aérea Argentina y la empresa estatal INVAP SE. En este artículo se reseña la historia de la política de radarización en el país, se explica el contexto en el cual esta política pública incorporó el desarrollo nacional de tecnología radar a partir del año 2004 y se presenta su implementación por distintos gobiernos hasta el año 2018. Palabras claves: Políticas tecnológicas, Política de control de trafico aéreo; Politica de radarización SINVICA

\section{National radar production: expression of a possible technological sovereignty}

Abstract: Argentina is a peripheral country, dependent on foreign powers in issues related to many knowledge-intensive technologies, even though, throughout its history has managed to reach partial sovereignty in others, such as the nuclear field. Regarding the air traffic and air space surveillance and control, the technological sovereignty was achieved, in a dependent context, after more than 50 years of learning, and as result of the joint efforts between the Argentinian Air Force and the stateowned company INVAP SE. The article reviews the history of air traffic control and surveillance policy in the country, explains the context in which this public policy incorporated the national development of radar technology since 2004, and presents its implementation by different governments until 2018. Key words: Technology policies, radar policy, Air traffic control policy, SINVICA

\section{Produção nacional de radares: expressão de uma soberania tecnológica possível}

Resumo: Argentina é um país periférico, dependente de poderes estrangeiros em questões relacionadas a muitas tecnologias intensivas em conhecimento, mas que ao longo de sua história conseguiu ser soberana em outras, como, por exemplo, no campo nuclear. Em relação ao controle de tráfego e ao espaço aéreo, a soberania tecnológica foi alcançada após mais de 50 anos de aprendizado em um contexto de dependência, graças à interação entre a Força Aérea Argentina e a empresa nacional INVAP SE. O artigo revisa a história da política de radarização no país; explica o contexto em que esta política pública incorporou o desenvolvimento nacional da tecnologia de radar a partir de 2004; e apresenta sua implementação pelos diferentes governos até 2018. 
| Ciencia, Tecnología y Política | Año 2 | No22 | Enero-Junio 2019 | ISSN 2618-3188 | www.revistas.unlp.edu.ar/CTyP |

Palavras-chave: Políticas de tecnologia; Política de controle de tráfego aéreo; SINVICA

\section{Introducción: historia de los radares y de su utilización en Argentina}

La tecnología radar permite detectar objetos por medio de ondas de radio y obtener información relevante sobre ellos. Se trata de una tecnología dual utilizada con diversos fines que abarcan usos en meteorología, radio astronomía, prospección y análisis de suelos, navegación marítima, aviación comercial y defensa. En este trabajo nos centraremos en estos dos últimos campos de aplicación, particularmente en sus usos para el control del tráfico aéreo y la vigilancia y control del espacio aéreo.

Los radares pueden clasificarse tomando en cuenta si la información se obtiene o no con la colaboración de los objetos detectados. Los radares primarios, utilizados con fines de defensa y control del espacio aéreo, no requieren "colaboración" del objeto detectado para obtener información acerca de su distancia al radar, acimut respecto a éste y/o altura. Por el contrario, los radares secundarios, utilizados también en control de tránsito aéreo y defensa, constan de un emisor terrestre que envía pulsos codificados que son respondidos por un artefacto llamado transponder, ubicado a bordo del avión, que da información sobre el mismo. Estos radares derivan del dispositivo Identification Friend or Foe diseñado durante la Segunda Guerra Mundial a fin de discernir en la totalidad de los ecos recibidos por los radares primarios, cuáles correspondían a aviones amigos y cuáles no.

Si bien existen antecedentes como los experimentos de Popov en 1897, o la patente del "Telemobiloskop" (un artefacto que permitía detectar barcos por medio de ondas de radio) de Hülsemeyer de 1904, el radar es un artefacto que fue desarrollándose en diversos lugares del mundo, durante la década de 1930, gracias a la pre-existencia de una base de conocimientos vinculados a la física y la ingeniería².

En ese momento, en cada país que incursionó en desarrollo de radares (tales como Alemania, EEUU, Francia, Holanda Japón, el Reino Unido, o la ex URSS) existieron factores que influyeron en la conformación de esta nueva tecnología. Entre estos, pueden mencionarse la motivación geopolítica para financiar el desarrollo, particularmente teniendo en cuenta la actitud ofensiva o defensiva frente al ambiente beligerante previo a la Segunda Guerra Mundial, el grado de apoyo gubernamental o la forma de utilización de la información obtenida por los radares. Es por ello que pese a que se lo ha señalado como un "desarrollo tecnológico simultáneo" (Süskind, 1994), surgieron diversos estilos socio-técnicos vinculados al radar que influyeron no solamente en su diseño, sino también en su forma de utilización, así como de la información obtenida con estos dispositivos (Quiroga, 2018b).

La Argentina fue un país pionero en Latinoamérica en lo referente a la utilización de radares para el control del espacio aéreo. Los primeros fueron adquiridos al Reino Unido en el año 1948 y comenzaron a ser operados por la Fuerza Aérea Argentina (FAA) en 1953, en torno a la ciudad de Buenos Aires. Estos radares fueron distribuidos en siete "estaciones radar" y constituían un sistema de alerta temprana ante potenciales incursiones aéreas hostiles. Sin embargo, a diferencia de lo que ocurrió -por ejemplo- con el desarrollo de la industria aeronáutica, donde el diseño y desarrollo nacional de aviones fue una actividad realizada

\footnotetext{
${ }^{1}$ La Real Academia Española define "acimut" como "el ángulo que con el meridiano forma el círculo vertical que pasa por un punto de la esfera celeste o del globo terráqueo".

2Para una revisión detallada de la historia del radar en el mundo puede consultarse Brown (1999).
} 
durante varias décadas (y en la cual se alcanzó un relativo éxito³) las actividades de l+D+i de tecnología radar nacional fueron actividades postergadas, pese a algunos desarrollos incipientes como el reemplazo de dos antenas radar a comienzos de la década de 1950 y a la orientación de las políticas industriales hacia la sustitución de importaciones.

Los avances referentes a la tecnología aeronáutica que se realizaron en los países centrales luego de finalizada la Segunda Guerra Mundial determinaron, consecuentemente, que la tecnología radar también tuviese que evolucionar hacia sistemas más complejos. Por este motivo, sumado a que los primeros radares operados por la FAA eran bastante rudimentarios en sus prestaciones y que se daba una reiterada falta de disponibilidad de repuestos, durante las décadas de 1960 y 1970 se adquirieron sucesivamente nuevos equipamientos y se reemplazó aquellos pre-existentes ${ }^{4}$. En todos estos casos se recurrió a proveedores extranjeros, realizando compras aisladas de diversos radares.

A fines de la década de 1970 la FAA impulsó el primer plan integrado de radarización, conocido como Proyecto Sistema Integrado de Control del Espacio Aéreo (SICEA). Dicho proyecto tenía como uno de sus objetivos generar un cambio "en el concepto operativo" de la vigilancia y control aeroespacial, dado que integraría la información radar de Tránsito Aéreo Civil con la concerniente a defensa. Sin embargo, debido el alto costo del mismo, debió dejarse de lado. En esa época, también se adquieren nuevos radares móviles (Westinghouse AN TPS 43) a los EEUU y se adquirió el primer radar para Control de Tráfico Aéreo (comprado a la firma francesa Thomson). Este último se emplazó en el Aeropuerto de Ezeiza, Buenos Aires, y constituyó el inicio de la radarización para el control del tránsito aerocomercial. Esta política continuó con la instalación de radares en las ciudades de Córdoba (1986); Mendoza (1993), Paraná (1996) y Mar del Plata (1997) adquiridos a la empresa italiana Alenia.

Por último, es necesario incorporar a este recuento la mención al Plan Nacional de Radarización (PNR) que fue impulsado, durante el gobierno de Menem en el año 1996, por medio del Decreto 145/96 que autorizaba al Ministerio de Defensa a llamar a licitación nacional e internacional para la compra de un sistema llave en mano que integrara el control de tráfico aéreo con el control del espacio aéreo. Sin embargo, el PNR no llegó a ejecutarse por diversas causas que incluyeron irregularidades en los términos de la licitación y acusaciones cruzadas entre las empresas participantes.

A lo largo de estos primero 50 años, Argentina se constituyó como un país dependiente en materia de tecnología radar (Quiroga y Aguiar, 2016). Esta dependencia tecnológica implicaba que la capacidad de compra del Estado se utilizara para comprar tecnología foránea con el consecuente gasto de divisas que ello supone. Sin embargo, y tal como sugería Jorge Sábato (2014 [1980]), en el marco de esta dependencia tecnológica en el seno de la FAA se generaron paulatinamente procesos de aprendizaje de saberes relacionados al radar. Éstos se referían inicialmente a cuestiones operativas vinculadas a la utilización y mantenimiento de radares y, con el tiempo, a cuestiones más ligadas al análisis y evaluación de tecnolo-

\footnotetext{
${ }^{3}$ Véase, por ejemplo, los trabajos de Lalouf (2005) o Artopoulos (2012). Respecto a los logros alcanzados y su comparación con la industria aeronáutica brasilera se puede consultar Hira y De Oliveira (2007).

${ }^{4}$ Para una referencia más detallada puede consultarse González (2014) y Quiroga y Aguiar (2016).
} 
gías, por ejemplo de propuestas de pliegos técnicos de licitaciones así como a la generación de planes integrales de radarización. Estos saberes permitieron concretar gradualmente compras con mejor capacidad de decisión sobre la tecnología adquirida y, posteriormente, constituyeron un insumo necesario para generar la base de conocimiento para su desarrollo nacional.

\section{INVAP, la FAA y el desarrollo de radares en Argentina}

El estado general de la radarización en Argentina al inicio del siglo XXI era aceptable en términos de control del tráfico aéreo (teniendo en cuenta el flujo de aeronaves que circulaba y la cobertura radar de las aerovías), pero precario en lo referente a control del espacio aéreo (defensa). Los radares de control de tráfico aéreo cubrían el centro del país, y se encontraban ubicados en las ciudades antes mencionadas, brindando cobertura a un corredor que concentraba, aproximadamente, el 75\% del tránsito aéreo comercial. Respecto al control del espacio aéreo, los cinco radares primarios móviles, cuyo estado de mantenimiento era ya de por sí crítico por falta de fondos, se vio agravado a partir del momento en que la empresa fabricante comunicó a la FAA la discontinuación de repuestos (Quiroga, 2018a).

Esta situación se produjo en un contexto signado por la aparición de nuevas problemáticas vinculadas a la seguridad y defensa en las cuales el radar tiene un rol preponderante: se instala, en la agenda mundial, el terrorismo como problema y la necesidad de incrementar los esfuerzos en la lucha contra el narcotráfico. Luego de los ataques terroristas en Nueva York, el 11 de Septiembre del 2001, se generó en los mandos superiores de la FAA la preocupación respecto a la falta de recursos, particularmente en lo referente al control y vigilancia del espacio aéreo, en pos de proteger potenciales objetivos de este tipo de ataques, como por ejemplo las centrales nucleares o sedes gubernamentales. Respecto al narcotráfico, se reconocía la necesidad de un mayor control de las fronteras, aspecto en el que la detección de tránsito aéreo irregular (TAI) era muy limitada. De esta manera, en el seno de la FAA comenzó a surgir, la necesidad de analizar alternativas para dotarse de capacidad operativa en vigilancia y control del espacio aéreo a fin de hacer frente al nuevo escenario existente.

La conjunción entre capacidades operativas y las nuevas problemáticas que se enfrentaban, crearon las condiciones para producir un cambio en la orientación de la política de radarización argentina, mediante la incorporación del desarrollo nacional de esta tecnología. Sin embargo, no alcanzaba con contar con las capacidades desarrolladas en el cuerpo de radaristas de la FAA (vinculadas principalmente al uso, mantenimiento y análisis técnico para definir pliegos y posterior evaluación de ofertas de licitaciones) para encarar el diseño y fabricación nacional de radares. Se requería también el dominio tecnológico y la expertise técnica en la cual basarse para diseñar y fabricar radares (Quiroga, 2017).

Durante el año 2002 la FAA comenzó a interactuar con la empresa rionegrina INVAP SE ${ }^{5}$ que, desde 1998,

\footnotetext{
5 INVAP SE es una empresa, propiedad del gobierno de la provincia de Río Negro, dedicada al desarrollo de bienes tecnológicos como reactores nucleares y satélites, entre otros. Véase Thomas, Versino y Lalouf (2008). En este mismo número, ver el artículo "Un análisis de la tecnopolítica aeroespacial argentina" de Sabando, Sarmiento y Hough.
} 
había sido contratada por la Comisión Nacional de Actividades Espaciales (CONAE) para diseñar algunos componentes y realizar la integración de dos de los cuatro satélites SAO-COM, cuya principal carga útil sería un radar de apertura sintética (SAR) 6 . Adicionalmente, INVAP se encontraba en una etapa singular, en la cual la dirección de la empresa buscaba enfocarse en generar negocios en áreas nuevas recurriendo para ello al aprovechamiento de las capacidades existentes. En cuanto a la gestión de proyectos, se hallaban en plena ejecución de las obras del reactor OPAL, en Australia, y por lo tanto INVAP contaba con flujos de fondos provenientes del extranjero para financiar la generación de nuevos proyectos. En ese contexto, a comienzos del año 2003, la empresa se pone en contacto con la FAA para ofrecer el desarrollo de radares para los aviones Pucará. Si bien este ofrecimiento no generó ninguna venta, el vínculo, en cambio, sirvió para que algunos oficiales de la FAA, vinculados a la vigilancia y control aeroespacial, tomaran conocimiento de que INVAP estaba desarrollando componentes de radares SAR, en un contexto en el que la capacidad operativa de la FAA en cuestiones vinculadas a control del espacio aéreo era limitada (Quiroga, 2017).

Cabe destacar que el hecho de que una empresa nacional proveyera radares a la FAA, además de no tener antecedentes en el país, resultaba beneficiosa para ambas partes. Desde la perspectiva de la FAA, porque solucionaba la falta de cobertura radar en aerovías que no contaban con ella y, en un futuro, permitiría eliminar la dependencia externa en la provisión de repuestos. Desde la perspectiva de INVAP, el encargo constituía la oportunidad de aplicar capacidades y conocimientos en el desarrollo de una nueva tecnología, ampliando su cartera de productos y diversificándola, puesto que hasta ese momento las principales áreas de negocios se vinculaban a las actividades nuclear y satelital (Quiroga, 2018a). De esta manera, el desarrollo de tecnología radar en Argentina permitía reducir la dependencia tecnológica, ahorrar divisas, generar puestos de trabajo calificados y desarrollar capacidades nacionales en una tecnología con alto valor agregado susceptible posteriormente de ser exportada.

\section{Política pública de radarización y fabricación de radares entre 2003 y 2019}

En marzo de 2003 la FAA e INVAP firmaron un acuerdo marco para el diseño y fabricación de un prototipo de radar de control de tránsito aéreo, el Radar Secundario Monopulso Argentino (RSMA) y en abril de ese año se firma un contrato (también entre FAA e INVAP) ad referéndum del ministerio de Defensa y la Jefatura de Gabinete para la fabricación de una serie de 10 RSMAs. Respecto al marco legal de la política pública de radarización cabe mencionar que el desarrollo de radares ya estaba en marcha, aunque aún seguía vigente el PNR de 1996, y en octubre de 2004 el Presidente Kirchner firma el Decreto 1407/04, por el cual se creaba el Sistema Nacional de Vigilancia y Control del Aeroespacio (SINVICA).

Este sistema integra la información de radares para control del espacio aéreo con los de control del tránsito

\footnotetext{
${ }^{6}$ Los satélites SAO-COM, son parte de un proyecto colaborativo entre la CONAE y la Agencia Espacial Italiana (Agenzia Spaziale Italiana, ASI). Entre ambas agencias se conformaría una constelación de satélites llamada SIASGE (Sistema Italo-Argentino de Satélites para Gestión de Emergencias), integrada por dos constelaciones de dos satélites argentinos SAO-COM (Satélite Argentino de Observación con Microondas) 1 y 2, y los sátelites denominados $1 \mathrm{~A}, 1 \mathrm{~B}, 2 \mathrm{~A}$ y $2 \mathrm{~B}$ respectivamente, y una constelación de cuatro satélites italianos COSMO-SkyMed. Respecto a los satélites argentinos cabe mencionarse que el primero (1A) fue puesto en órbita durante 2018, el segundo (1B) se haya en integración CONAE (2019). Para un mayor detalle sobre la participación de INVAP en el diseño y fabricación de radares radares SAO para las misiones SAO-COM y su vínculo con el desarrollo posterior de radares secundarios y primarios puede consultarse Quiroga y Aguiar (2016).
} 
aéreo y prevé cuatro componentes constitutivos del sistema: radares (primarios y secundarios), aviones interceptores, sistemas de procesamiento e integración de la información y sistemas de comunicaciones. Su objetivo es «permitir al Estado argentino efectuar el control de todos los movimientos aéreos en el espacio aéreo de jurisdicción nacional [...] contribuyendo de esa manera al cumplimiento de las tareas de defensa aeroespacial y a prestar un eficiente servicio de tránsito aéreo» (Decreto 1407/04). Un aspecto destacable del Decreto, es el ya mencionado giro que supone respecto a las normas anteriores al estipularse que, en la medida de lo posible, deberá utilizarse «mano de obra técnica y capacidades de la industria nacional para el diseño, desarrollo, ensamblado, construcción, prueba, operación y mantenimiento del sistema», idea que iría afianzándose a lo largo del tiempo, tal como se desprende del énfasis creciente que fue otorgándosele a este tipo de consideración en normas y contratos complementarios.

El impulso dado por los gobiernos kirchneristas (2003-2015) al diseño y fabricación de radares basado en el mencionado Decreto 1407/04 se materializó en forma paulatina: en noviembre de 2006 el Ministerio de Defensa aprobó el contrato firmado entre la FAA e INVAP, para la provisión de un prototipo del RSMA y la posterior fabricación e instalación de una serie de diez de estos radares secundarios? ${ }^{7}$. Luego, en 2008 se encargó a INVAP la provisión de un prototipo de radar primario (Radar Primario Argentino 3D de Largo Alcance o RPA3D-LA). Posteriormente, el 17 de septiembre de 2010, el Ministerio de Defensa aprobó el suministro de seis radares RPA3D-LA (Serie 1). También durante 2010, la Administración Nacional de Aviación Civil (ANAC) dependiente del Ministerio de Planificación Federal Inversión Pública y Servicios (MPFIPyS) acuerda la compra a INVAP de once RSMAs adicionales (Serie 2). De esta manera, hacia fines de 2014 veintidós RSMAs fabricados por INVAP se habían instalado a lo largo y ancho del país. En la Figura 1 se muestra la cobertura radar para control de tráfico aéreo en 2000 y en 2014, con la utilización de RSMAs.

En noviembre de 2015, cuando se habían instalado dos de los seis RPAs previstos (además del prototipo operativo) de la Serie 1, se firmó un nuevo contrato con INVAP para la provisión de 6 adicionales (Serie 2). En diciembre de 2015, con la llegada al gobierno de la alianza Cambiemos encabezada por Macri, la coalición gobernante decidió deliberadamente disminuir sensiblemente la inversión pública en ciencia y tecnología y el apoyo estatal a la industria nacional, lo cual se efectivizó de diversas maneras. En el caso de la empresa INVAP, esto impactó por un lado, por la cancelación de contratos previamente firmados para desarrollo y adquisición -por parte del Estado nacional- de bienes tecnológicos que ya estaban en ejecución. Entre ellos el sistema de satélites livianos SARE, el satélite de telecomunicaciones ARSAT 3 y el Sistema Aéreo Robótico Argentino, SARA. Por otro lado en la postergación de los pagos de contratos de proyectos que continuaron su ejecución (de la Vega, 2018). Si bien gran parte de la radarización para control de tránsito aéreo ya estaba avanzada, aún quedaban contratos pendientes de ejecución en lo referido tanto a fabricación e instalación de radares primarios como al mantenimiento del conjunto de los radares secundarios instalados por INVAP.

En diciembre de 2015 se estaba finalizando la instalación del tercer RPA de la Serie 1 en San Pedro, Misiones. INVAP también aguardaba novedades para la firma de un contrato de modernización de cuatro radares móviles AN TPS 43 de la FAA. Sin embargo a partir de ese momento el ritmo de producción de los RPAs debió adaptarse a los tiempos de entregas re-pactados, como consecuencia de las "condiciones presupuestarias" (INVAP SE, 2017) del cliente, en este caso, el Ministerio de Defensa. Los últimos tres RPAs de la serie 1

\footnotetext{
7 INVAP trabajó a riesgo propio en el diseño y fabricación del prototipo del RSMA durante más de 2 años. Esto fue posible gracias a la disponibilidad de fondos que tenía la empresa por el contrato por el reactor OPAL de Australia.
} 

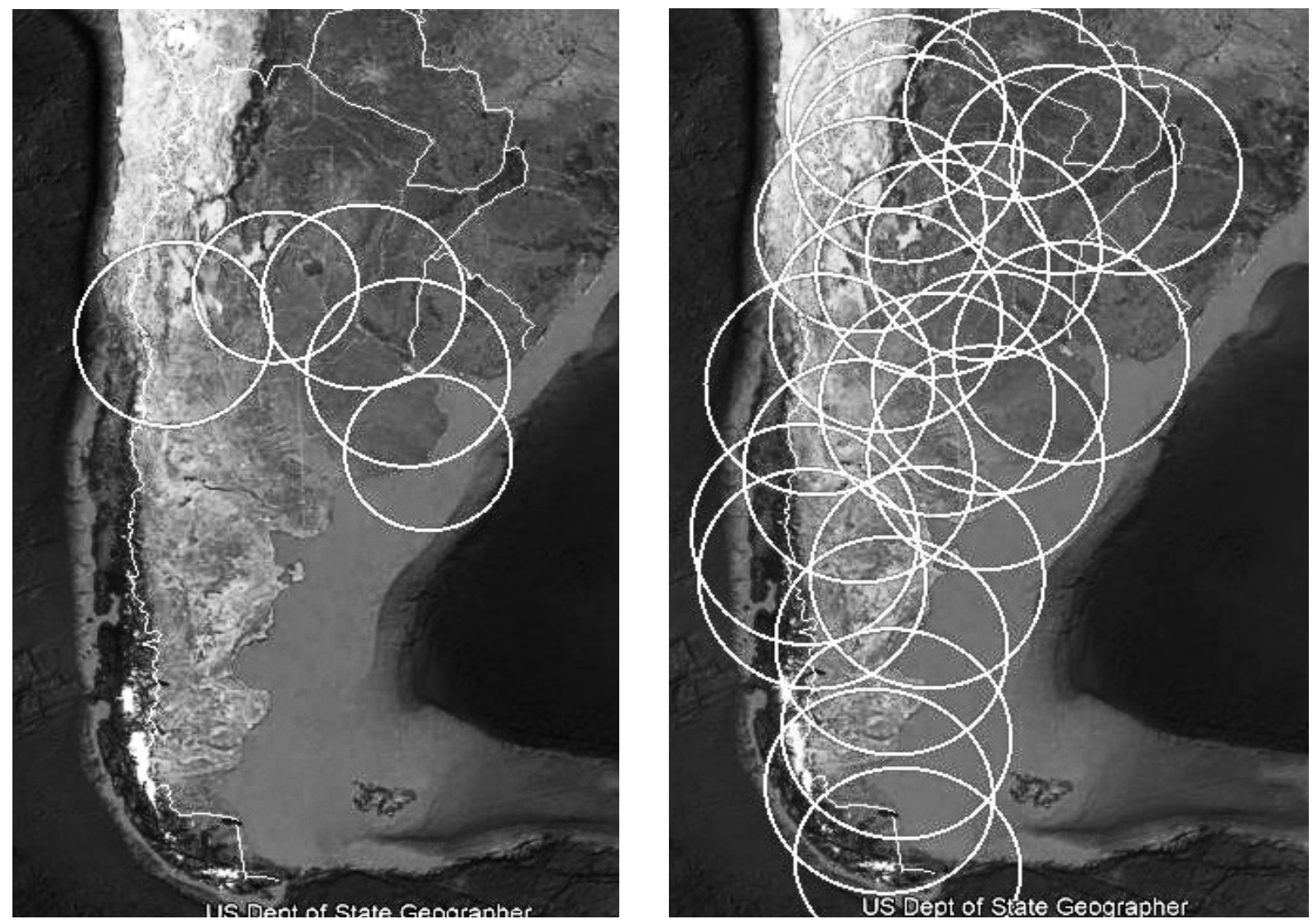

Figura 1. Cobertura de radares de control de tránsito aéreo en Argentina en 2000 (a) y en 2014 (b).Fuente: Quiroga (2017). Mapas de Google earth Pro.

estuvieron fabricados y almacenados en depósitos de INVAP desde mediados de 2016, informando la empresa en junio de 2018 que se habían comenzado las obras para construir la base de uno de ellos (el cuarto) en Pirané, Formosa, y en noviembre empezarían los trabajos de emplazamiento del radar de Villaguay. El restante, sexto de la Serie 1, aún esperaba la definición del lugar de su emplazamiento definitivo. Respecto a la Serie 2 de RPAs, hasta junio de 2018 se había fabricado el primer radar y se trabajaba en su certificación (INVAP SE, 2018; 2017; 2016).

Adicionalmente a la fabricación de RPAs, cabe mencionarse que INVAP firmó en noviembre de 2017 dos nuevos contratos con la FAA. El primero referido a la modernización de dos radares AN TPS 43, uno de los cuales ya había tenido una actualización previa, tal como ya se ha mencionado. El otro contrato era por la fabricación de una unidad, también modernizada, en este caso del Radar Argentino de Alcance Mediano ${ }^{8}$ (RAME), llamada RAM2. Ambos trabajos fueron realizados en "tiempo record" para que pudieran ser utilizados en tareas de vigilancia y control del espacio aéreo durante la cumbre del G-20 realizada en Buenos Aires en 2018 (INVAP SE, 2018). Finalmente, en enero de 2019 la Empresa Argentina de Navegación Aérea (EANA), contrató a INVAP la provisión e instalación de un RSMA en la ciudad de Paraná, provincia de Entre Ríos. El nuevo radar incorporará tecnología ADS-B9 (INVAP SE, 2019).

\footnotetext{
${ }^{8}$ El RAME es un subproducto del desarrollo del RPA3D-LA, actualmente utilizado por la FAA y que INVAP vendió al Ministerio de Defensa, evitando la compra de tecnología extranjera. Véase Quiroga (2017).

${ }^{9}$ Esta tecnología permite "precisar al control aéreo en ruta la posición de las aeronaves mediante la información que los sistemas de éstas emiten periódicamente a través de sus equipos de navegación satelital".
} 


\section{Conclusiones: Las políticas públicas como basamento de la soberanía tecnológica}

Al finalizar el siglo XX la Argentina se hallaba en una situación delicada respecto al control de su espacio aéreo y la radarización para control del tránsito aéreo era limitada, situación que se daba en el marco de una absoluta dependencia tecnológica en la materia. Con la llegada del nuevo siglo, este escenario comenzó a cambiar como consecuencia de una serie de acontecimientos, entre los que se puede mencionar el pronóstico de aumento del tráfico aéreo comercial, la creciente amenaza del "terrorismo", la necesidad de incrementar la lucha contra el narcotráfico y la necesidad de lidiar con la falta de repuestos de radares extranjeros.

En ese marco, durante 2004 la FAA e INVAP acordaron el diseño y fabricación de un prototipo de radar secundario, se creó el SINVICA y, posteriormente, en este marco se avanzaría con la fabricación de dos series de 10 y 11 radares RSMA, respectivamente. Estos radares han permitido incrementar la seguridad en los vuelos que transitan rutas aerocomerciales en Argentina, al tiempo que disminuye la distancia mínima entre dos vuelos, permitiendo ahorrar dinero en combustible a las aerolíneas al optimizar la altura de los aviones en vuelo y disminuir las emisiones contaminantes. También ha posibilitado contar con la capacidad de gestionar un incremento en el tráfico aéreo en nuestro país, y por ende también fue un factor importante para sentar las bases de expansión del mercado aerocomercial doméstico.

También gracias a esta decisión política orientada al desarrollo nacional de tecnologías, en 2008 se inició el desarrollo, mucho más ambicioso, de un radar primario, el RPA, que llevaría a la firma de dos contratos, por una serie de seis radares cada uno, la venta de un sub-producto del proceso de diseño y desarrollo del RPA, el RAME, y la modernización de radares AN TPS 43 de la FAA.

El caso del SINVICA permite mostrar que, pese a la dependencia tecnológica, es posible desarrollar capacidades que con el tiempo, la complementación entre distintos tipos de organizaciones, y un apoyo político adecuado pueden constituir insumos para lograr tecnologías soberanas. Este tipo de tecnologías son generadoras de nuevas capacidades, conllevan la creación de puestos de trabajo calificados, disminuyen la dependencia, son susceptibles de generar ahorros de divisas y, eventualmente, exportarse como bienes de alto valor agregado. Es por ello que las políticas tecnológicas que apuntan a lograr soberanía deberían tener continuidad en el tiempo, transformándose en políticas de Estado. En este sentido, el caso de la radarización en Argentina, constituye una política pública heredada del kirchnerismo, que si bien no ha sido dejada totalmente de lado a partir de la llegada al poder de la alianza Cambiemos, se vio afectada por diversas medidas como la cancelación de contratos y la ralentización de los pagos al proveedor, que llevaron a diferir entregas de radares primarios y de firma de convenios de mantenimiento a largo plazo.

Por otro lado, en el marco de políticas públicas de corte neoliberal, a comienzos de 2017 el gobierno de Macri desreguló aspectos tarifarios del mercado aerocomercial, en la llamada "revolución de los aviones", con el objetivo de duplicar la cantidad de pasajeros transportados en 2019, incrementar la cantidad de empresas que realizan actividades de transporte aerocomercial en el país y modernizar aeropuertos. Para que todo esto sea posible, es necesario contar con una adecuada infraestructura, en la cual la radarización en- 
carada en el marco del SINVICA es clave para dotar de seguridad al tráfico aéreo, y permitir su incremento. Esto permite ejemplificar el hecho de que las políticas públicas constituyen "capas geológicas" superpuestas (Lavarello y Sarabia, 2015). De esta manera, aún para poder pensar en llevar a cabo la desregulación del mercado aerocomercial con vistas a su expansión, más allá de lo discutible de esta decisión, fue condición sine qua non haber contado previamente con la inversión estatal en radarización descripta en este artículo.

Finalmente, el caso analizado pone en evidencia que más allá de la generación de capacidades, el impacto socio económico de alcanzar soberanía tecnológica en ciertas áreas constituye un aspecto fundamental a tener en cuenta para lograr un crecimiento y desarrollo equitativo y soberano de nuestro país. Se hace necesario entonces generar espacios de discusión para que, tanto la sociedad en general, como sus instituciones y sobre todo sus estructuras políticas y sociales puedan comprender la importancia de este tipo de políticas públicas científico-tecnológicas a fin de transformarlas en políticas de Estado, más allá de la orientación de cada gobierno de turno.

\section{Bibliografía}

Artopoulos, A. (2012). Tecnología e innovación en países emergentes. La aventura del Pulqui II (19471960). Buenos Aires: Lenguaje Claro Editora.

Brown, L. (1999). A radar history of World War II - Technical and military imperatives. Bristol: Institute of Physics Publishing.

CONAE (2019). SAOCOM. Recuperado el 28 de Febrero de 2019 de http://www.conae.gov.ar/index. php/espanol/misiones-satelitales/saocom/objetivos

de la Vega, C. (2018). ¿Por qué nos importa INVAP? Recuperado el 28 de Febrero de 2019, de http:// www.unsam.edu.ar/tss/por-que-nos-importa-invap/

González, O. F. (2014). Vigilancia y Control Aéreo en Argentina. Buenos Aires: Ediciones Argentinidad.

Hira, A., y De Oliveira, L. (2007). Take off and crash: lessons from the diverging fates of the Brazilian and Argentine aircraft industries. Competition and Change, 11(4), 329-347.

INVAP SE. (2016). Estados Financieros correspondientes al ejercicio finalizado el 30 de Junio de 2016. San Carlos de Bariloche.

INVAP SE. (2017). Estados Financieros correspondientes al ejercicio finalizado el 30 de Junio de 2017. San Carlos de Bariloche.

INVAP SE. (2018). Estados Financieros correspondientes al ejercicio finalizado el 30 de Junio de 2018. San Carlos de Bariloche.

INVAP SE. (2019). EANA e INVAP firmaron un contrato para la instalación de un radar de última generación en el aeropuerto de Paraná. Consultado el 13 de Febrero de 2019, de http://www.invap.com.ar/ es/la-empresa/sala-de-prensa/novedades/1649-eana-e-invap-firmaron-un-contrato-para-la-instalacion-de-un-radar-de-ultima-generacion-en-el-aeropuerto-de-parana.html

Lalouf, A. (2005). Construcción y Desconstrucción de un 'caza nacional': análisis socio-técnico de la experiencia de diseño y producción de los aviones Pulqui I y Pulqui II (Argentina 1946/1960). Tesis de 
Maestría. Universidad Nacional de Quilmes - Maestría en Ciencia, Tecnología y Sociedad.

Lavarello, P., y Sarabia, M. (2015). La política industrial en la Argentina durante la década de 2000. Santiago de Chile: CEPAL-ONU.

Quiroga, J. M. (2017). Desarrollo de radares secundarios y primarios en la Argentina (2003-2015). Un análisis desde el enfoque de coaliciones de causa y las capacidades organizacionales. Tesis de Maestría. Universidad Nacional de Río Negro.

Quiroga, J. M. (2018a). Políticas públicas, trayectorias institucionales y desarrollo tecnológico nacional. Los primeros sesenta años de tecnología radar en la Argentina. In D. Aguiar, M. Lugones, J. M. Quiroga, y F. Aristimuño (Eds.), Políticas de ciencia, tecnología e innovación en la Argentina de la posdictadura (pp. 79-104). Viedma: Editorial UNRN.

Quiroga, J. M. (2018b). Primeros desarrollos de tecnología radar en los principales beligerantes de la II Guerra Mundial. Un análisis desde la perspectiva Ciencia, Tecnología y Sociedad. Ciencia, Docencia y Tecnología, 29(57), 36-59.

Quiroga, J. M., y Aguiar, D. (2016). Abriendo la "caja negra" del radar. Las políticas de radarización para uso civil y de defensa en Argentina entre 1948 y 2004. H-Industri@, 10(19), 71-100.

Sábato, J. (2014 [1980]). Desarrollo tecnológico en América Latina y el Caribe. En J. Sábato; S. Harriage y D. Quilici (Eds.), Estado, política y gestión de la Tecnología. Obras escogidas (1962-1983) (pp. 201-218). San Martín: UNSAM Edita.

Süsskind, C. (1994). Radar as case study in simultaneous invention. In O. Blumtritt, H. Petzold, \& W. Aspray (Eds.), Tracking the history of radar (pp. 237-246). Piscataway, New Jersey: IEEE Rutgers Center for the History of Electrical Engineering and Deutsches Museum.

Thomas, H., Versino, M., y Lalouf, A. (2008). La producción de tecnología nuclear en Argentina: el caso de la empresa INVAP. Desarrollo Económico, 47(188), 543-575 